TRANSACTIONS OF THE

AMERICAN MATHEMATICAL SOCIETY

Volume 353, Number 1, Pages 247-267

S 0002-9947(00)02743-4

Article electronically published on September 13, 2000

\title{
SPECTRAL THEORY AND HYPERCYCLIC SUBSPACES
}

\author{
FERNANDO LEÓN-SAAVEDRA AND ALFONSO MONTES-RODRÍGUEZ
}

\begin{abstract}
A vector $x$ in a Hilbert space $\mathcal{H}$ is called hypercyclic for a bounded operator $T: \mathcal{H} \rightarrow \mathcal{H}$ if the orbit $\left\{T^{n} x: n \geq 1\right\}$ is dense in $\mathcal{H}$. Our main result states that if $T$ satisfies the Hypercyclicity Criterion and the essential spectrum intersects the closed unit disk, then there is an infinite-dimensional closed subspace consisting, except for zero, entirely of hypercyclic vectors for $T$. The converse is true even if $T$ is a hypercyclic operator which does not satisfy the Hypercyclicity Criterion. As a consequence, other characterizations are obtained for an operator $T$ to have an infinite-dimensional closed subspace of hypercyclic vectors. These results apply to most of the hypercyclic operators that have appeared in the literature. In particular, they apply to bilateral and backward weighted shifts, perturbations of the identity by backward weighted shifts, multiplication operators and composition operators. The main result also applies to the differentiation operator and the translation operator $T$ : $f(z) \rightarrow f(z+1)$ defined on certain Hilbert spaces consisting of entire functions. We also obtain a spectral characterization of the norm-closure of the class of hypercyclic operators which have an infinite-dimensional closed subspace of hypercyclic vectors.
\end{abstract}

\section{INTRODUCTION}

A bounded operator $T$ on a Hilbert space $\mathcal{H}$ is said to be cyclic if there is a vector $x \in \mathcal{H}$ such that the orbit $\left\{T^{n} x\right\}_{n \geq 1}$ has dense linear span. If this is the case, the vector $x$ is called a cyclic vector for $T$. If the orbit $\left\{T^{n} x\right\}_{n>1}$ is itself dense in $\mathcal{H}$, then $T$ is said to be hypercyclic. In this case the vector $x$ is called hypercyclic for $T$.

Each of the following classes of linear maps contains hypercyclic operators: backward and bilateral shifts [Ro, GS], Sa2 , translations and differentiation operators [CS], composition operators [BS1, BS2, multiplication operators GoS], perturbation of the identity by a weighted shift Sa2].

Interest in cyclic operators arises from the invariant subspace problem. In fact, it is easy to see that an operator $T$ has no non-trivial invariant closed subspace if and only if each non-zero vector is cyclic for $T$. It is not known if there is a bounded linear operator on a separable Hilbert space that does not have closed, non-trivial invariant subspaces. Similarly, an operator has no non-trivial closed invariant subset if and only if each non-zero vector is hypercyclic. Again, it is not known if there is an operator on Hilbert that does not have closed, non-trivial

Received by the editors April 14, 1997.

2000 Mathematics Subject Classification. Primary 47A16, 47A53; Secondary 47 B37.

Key words and phrases. Hypercyclic operator, hypercyclic vector, essential spectrum, essential minimum modulus, bilateral shift, backward shift, multiplier, composition operator, differentiation operator, translation operator. 
invariant subset. On Banach space the situation is different: Enflo [En] solved the invariant subspace problem, and Read [Re constructed on the space $\ell^{1}$ an operator without invariant closed subset.

Herrero [He3] and Bourdon [Bou] independently showed that every hypercyclic operator on Hilbert space has a (non-closed) dense invariant subspace that consists, except for the zero vector, entirely of hypercyclic vectors, thus completing earlier results that appeared in $[\mathrm{Be}], \mathrm{GoS}]$ and $[\mathrm{Pa}]$. As a consequence, the restriction of a hypercyclic operator to such a subspace gives an example of a bounded linear operator on a pre-Hilbert space with no proper, closed invariant subset.

There is also some work which complements Herrero's and Bourdon's result. Bernal and Montes $[\mathrm{BM}]$ proved that there is an infinite-dimensional closed vector space consisting, except for zero, of hypercyclic vectors for the translation operator in the space of entire functions with the topology of uniform convergence on compact subsets. This result has been extended to Banach spaces: The main result in $\mathrm{MO}$ ] gives sufficient conditions on a bounded linear operator defined on a Banach space which guarantee that there exists a whole infinite-dimensional Banach space which consists, except for zero, of hypercyclic vectors. This result was used to prove that certain composition operators acting on Hardy spaces have an infinite-dimensional closed subspace of hypercyclic vectors (see Theorem 3.1 in [Mo]). In strong contrast with Herrero's and Bourdon's result, there exist hypercyclic operators such that all closed subspaces of hypercyclic vectors for $T$ are finite dimensional - in particular, certain scalar multiples of the backward shift defined on $\ell^{2}$ (see [Mo, Theorem 3.4]). Therefore, the following question arises:

Question. Which hypercyclic operators have an infinite-dimensional closed subspace of hypercyclic vectors?

A partial solution to this question was given in $[\mathrm{LM}]$. Specifically, if an operator $T$ is a compact perturbation of the identity and satisfies the Hypercyclicity Criterion, then there is an infinite-dimensional closed subspace consisting, except for zero, of hypercyclic vectors for $T$. This is a somewhat surprising conclusion, because compact perturbations of the identity were not expected to be hypercyclic (see [CS], [HW], [Sa2]).

In Section 2 we state the main result, which answers the above question for all operators satisfying the Hypercyclicity Criterion. Then we apply the result to bilateral and backward weighted shifts, perturbation of the identity by a backward weighted shift, multiplication operators and composition operators. The main result also applies to the differentiation operator and the translation operator $T: f(z) \rightarrow$ $f(z+1)$ on certain Hilbert spaces consisting of entire functions. Thus we answer some of the questions posed in [LM].

Section 3 is devoted to proving that if an operator $T$ satisfies the Hypercyclicity Criterion and the essential spectrum intersects the closed unit disk, then there is an infinite-dimensional closed subspace consisting, except for zero, entirely of hypercyclic vectors. Section 4 is an expository section devoted to the concept of the essential minimum modulus. The essential minimum modulus is the most important technical concept in the following section. Section 5 is devoted to proving that if an operator is hypercyclic and the essential spectrum does not intersect the closed unit disk, then all closed subspaces of hypercyclic vectors are finite-dimensional. The essential minimum modulus will play a critical role in an argument that depends in an essential way on a deep theorem of Zemánek that relates asymptotic properties of the essential minimum modulus with the distance from zero to the left essential 
spectrum. We will close Section 5 with a characterization of the norm-closure of the hypercyclic operators which have an infinite-dimensional closed subspace of hypercyclic vectors.

The results of this work clarify the idea suggested in [LM] that "the less opportunity an operator has of being hypercyclic, the more opportunity it has of supporting an infinite-dimensional closed subspace of hypercyclic vectors".

Before proceeding further we would like to say that the authors are deeply indebted to Joel H. Shapiro, who has contributed a great deal of work and very sharp observations to improve this paper.

\section{Statement and applications}

Throughout this section $\mathcal{H}$ will denote a separable Hilbert space. We start by recalling some definitions from spectral theory (see [He1 Chapter 1]). Let $\mathcal{L}(\mathcal{H})$ denote the space of bounded linear operators which apply $\mathcal{H}$ into itself. The spectrum of an operator $T$ is $\sigma(T)=\{\lambda \in \mathbb{C}: T-\lambda$ is not invertible $\}$. The left spectrum of $T$ will be denoted by $\sigma_{l}(T)$. It is the set of complex numbers $\lambda$ such that $T-\lambda$ is not left invertible. The right spectrum $\sigma_{r}(T)$ is defined similarly.

If $\mathcal{K}(\mathcal{H})$ denotes the ideal of all compact operators acting on $\mathcal{H}$, then the Calkin algebra is the quotient space $\mathcal{L}(\mathcal{H}) / \mathcal{K}(\mathcal{H})$. If $T \in \mathcal{L}(\mathcal{H})$, then the canonical projection $\pi(T)$ onto $\mathcal{L}(\mathcal{H}) / \mathcal{K}(\mathcal{H})$ will be denoted by $\widetilde{T}$. The essential spectrum of $T$ is $\sigma_{e}(T)=\sigma(\widetilde{T})$. The left essential spectrum $\sigma_{l e}(T)$ and the right essential spectrum $\sigma_{r e}(T)$ are defined in the obvious way. Recall that $T \in \mathcal{L}(\mathcal{H})$ is called Fredholm if $\operatorname{ran} T$ is closed and the index ind $T=\operatorname{dim}(\operatorname{ker} T)-\operatorname{dim}\left(\operatorname{ker} T^{\star}\right)$ is finite. Atkinson's Theorem (see [Ha, p. 91]) asserts that $T$ is Fredholm if and only if $\tilde{T}$ is invertible in the Calkin algebra. Hence, $\rho_{F}(T)=\{\lambda \in \mathbb{C}: T-\lambda$ is Fredholm $\}$ (the Fredholm domain of $T$ ) is an open subset of $\mathbb{C}$ and $\sigma_{e}(T)=\mathbb{C} \backslash \rho_{F}(T)$.

We will rely heavily on the following theorem, which gives sufficient conditions for an operator $T$ to have an infinite-dimensional closed subspace of hypercyclic vectors for a bounded operator $T$ on a separable Banach space (see $\mathrm{Mo}$ Theorem 2.2 and the remarks following it]).

Theorem A. Let $T$ be a bounded linear operator on a separable Banach space $\mathcal{B}$. Suppose that there exists a sequence $\left\{n_{k}\right\}$ of positive integers, strictly increasing to $\infty$, corresponding to which there are

a1) a dense subset $X \subset \mathcal{B}$ such that $\left\|T^{n_{k}} x\right\| \rightarrow 0$ for every $x \in X$,

a2) a dense subset $Y \subset \mathcal{B}$ and a mapping $S: Y \rightarrow Y$ such that $T S=$ identity on $Y$, and $\left\|S^{n_{k}} y\right\| \rightarrow 0$ for every $y \in Y$, and

b) an infinite-dimensional closed subspace $\mathcal{B}_{0} \subset \mathcal{B}$ such that $\left\|T^{n_{k}} e\right\| \rightarrow 0$ for every $e \in \mathcal{B}_{0}$.

Then, there is an infinite-dimensional closed subspace $\mathcal{B}_{1}$ such that each $z \in \mathcal{B}_{1} \backslash\{0\}$ is hypercyclic for $T$.

It is known that an operator satisfying a1) and a2) for a sequence $\left\{n_{k}\right\}$ is hypercyclic, in fact, this is the result known as the Hypercyclicity Criterion. The Hypercyclicity Criterion was discovered by Carol Kitai in her 1982 Toronto dissertation [Ki]. This result was never published and it was rediscovered by Gethner and Shapiro [GS] in more generality and with a simpler proof. They used it to unify the proofs of theorems of Birkhoff [Bi], Seidel and Walsh SW], MacLane [Mc], and Rolewicz $[\mathrm{Ro}$, and to discover hypercyclic behavior in many other settings. Since 
then, the Hypercyclicity Criterion has figured prominently in subsequent studies to discover hypercyclic behavior [BS1, [BS2, GoS, [CS, [He3, [HW]. Theorem A states that the Hypercyclicity Criterion plus condition b) give a whole infinitedimensional closed subspace of hypercyclic vectors. From now on, for the sake of fluency we omit the phrase "except for zero". Although conditions a1) and b) might appear similar at first glance, they are quite the opposite of each other. Indeed, if property b) is satisfied for the whole sequence of natural numbers, then hypercyclic vectors cannot exist in $\mathcal{B}_{0}$.

Observe that the class of all operators having an infinite-dimensional closed subspace of hypercyclic vectors and the class of all operators satisfying property b) of Theorem A are invariant under similarity. Therefore, as Herrero did with the class of all hypercyclic operators in $\mathrm{He} 3$, these properties can be analyzed with the approximation machinery developed in AFHV], He1], [He2]. Usually, this machinery gives a characterization of the norm-closure of the operators satisfying some certain property. In a sense the next result, which is the main goal of this paper, tells us that we can do a little more.

Theorem 2.1. Let $T$ be a bounded linear operator on a separable Hilbert space $\mathcal{H}$ satisfying the Hypercyclicity Criterion. Then the following conditions are equivalents:

i) There is an infinite-dimensional closed subspace $\mathcal{H}_{1}$ such that each $z \in$ $\mathcal{H}_{1} \backslash\{0\}$ is hypercyclic for $T$.

ii) There exists a sequence of positive integers $\left\{n_{k}\right\}$ corresponding to which there is an infinite-dimensional closed subspace $\mathcal{H}_{0} \subset \mathcal{H}$ such that $\left\|T^{n_{k}} z\right\| \rightarrow 0$ for every $z \in \mathcal{H}_{0}$.

iii) There exists a sequence of positive integers $\left\{n_{k}\right\}$ corresponding to which there are an infinite-dimensional closed subspace $\mathcal{H}_{b} \subset \mathcal{H}$ and a constant $M>0$ such that $\left\|T^{n_{k}} z\right\| \leq M\|z\|$ for every $z \in \mathcal{H}_{b}$ and for every $k$.

iv) The essential spectrum of $T$ intersects the closed unit disk.

We stress here that in order to apply Theorem A it is necessary to verify conditions a) and b) on the same subsequence of positive integers. Observe that Theorem 2.1 does not need this requirement. Condition iii) is another improvement of Theorem A. From Theorem 2.1 we see that condition b) in Theorem A was quite sharp. Theorem 2.1 says that in some sense certain hypercyclic operators (including compact perturbations of the identity) are more hypercyclic than others.

Theorem 2.1 will be proved in Section 5. We devote the rest of the present section to applying the result to the most common classes of operators which are known to contain hypercyclic operators.

1. Bilateral weighted shifts. Assume now that $\mathcal{H}=\ell^{2}(\mathbb{Z})$. The operator $T$ is an (injective) bilateral (forward) weighted shift with respect to the canonical basis $\left\{e_{n}: n \in \mathbb{Z}\right\}$ if $T e_{n}=w_{n} e_{n+1}$, where the weight sequence $\left\{w_{n}: n \in \mathbb{Z}\right\}$ is a bounded subset of $\mathbb{C} \backslash\{0\}$ (without loss of generality we may assume that each $w_{n}$ is positive). Salas [Sa2] proved that a weighted shift with positive weight sequence $\left\{w_{n}\right\}$ is hypercyclic if and only if, given $\varepsilon>0$ and a positive integer $q$, there exists $n$ arbitrarily large such that

$$
\prod_{s=0}^{n-1} w_{s+j}<\varepsilon \quad \text { and } \quad \prod_{s=1}^{n} w_{j-s}>\frac{1}{\varepsilon} \quad(|j| \leq q) .
$$


In order to apply Theorem 2.1, let us show that a hypercyclic bilateral weighted shift always satisfies the Hypercyclicity Criterion. To verify conditions a1) and a2) of Theorem A we follow the lines of Gethner and Shapiro. We take $X=Y=$ $\operatorname{span}\left\{e_{n}\right\}$, and the operator $S$ will be the backward weighted shift defined on $\ell^{2}(\mathbb{Z})$ by

$$
S e_{n}=\frac{1}{w_{n-1}} e_{n-1} \quad(n \in \mathbb{Z}) .
$$

Clearly, $T S=$ identity on $\ell^{2}(\mathbb{Z})$. Observe that if the sequence $\left\{w_{n}\right\}$ is not bounded away from zero, then $S$ is not a bounded operator. But the Hypercyclicity Criterion copes as well with this more general situation. Let $\left\{\varepsilon_{k}\right\}_{k \geq 1}$ be any sequence of positive numbers decreasing to zero. Upon applying conditions (1) for each positive integer $k$ we can find a positive integer $n_{k}$ such that conditions (1) are satisfied for $\varepsilon=\varepsilon_{k}, q=k$ and $n=n_{k}$. The sequence required by the Hypercyclicity Criterion will be $\left\{n_{k}\right\}$. Therefore, if $x \in \operatorname{span}\left\{e_{n}\right\}$, then $x=\sum_{i=-k_{0}}^{k_{0}} \alpha_{i} e_{j}$, where $k_{0}$ is a positive integer and some $\alpha_{j}$ may be zero. So if $k \geq k_{0}$, we have

$$
\begin{aligned}
\left\|T^{n_{k}} x\right\| & =\left\|\sum_{j=-k_{0}}^{k_{0}} \alpha_{j}\left(\prod_{s=0}^{n_{k}-1} w_{s+j}\right) e_{j+n_{k}}\right\| \\
& =\left(\sum_{j=-k_{0}}^{k_{0}}\left|\alpha_{j}\right|^{2}\left(\prod_{s=0}^{n_{k}-1} w_{s+j}\right)^{2}\right)^{1 / 2} \\
& <\varepsilon_{k}\|x\|,
\end{aligned}
$$

which tends to zero as $k$ tends to infinity. On the other hand,

$$
\begin{aligned}
\left\|S^{n_{k}} x\right\| & =\left\|\sum_{j=-k_{0}}^{k_{0}} \alpha_{j} \frac{1}{\left(\prod_{s=1}^{n_{k}} w_{j-s}\right)} e_{j-n_{k}}\right\| \\
& =\left(\sum_{j=-k_{0}}^{k_{0}}\left|\alpha_{j}\right|^{2} \frac{1}{\left(\prod_{s=1}^{n_{k}} w_{j-s}\right)^{2}}\right)^{1 / 2} \\
& <\varepsilon_{k}\|x\|,
\end{aligned}
$$

which also tends to zero as $k \rightarrow \infty$.

The spectrum of a bilateral weighted shift $T$ is either an annulus or a disk, depending on whether $T$ is invertible or not (see [Shi Theorem 5, p. 67]). First, we suppose that the spectrum is a disk. Since bilateral weighted shifts have dense range and are injective, we find that $\operatorname{ran} T$ is not closed; otherwise $T$ would be invertible. Thus, the origin belongs to the essential spectrum and, therefore, the essential spectrum intersects the closed unit disk. Now suppose that the spectrum is an annulus. Having in mind that each component of the spectrum of a hypercyclic operator must intersect the unit circle (see [Ki Theorem 2.8]), we find that the boundary of the annulus intersects the closed unit disk. On the other hand, for any operator nonisolated points in the boundary of the spectrum belong to the essential spectrum (see [Co, Chapter XI, Theorem 5.3]). Therefore, we find that the essential spectrum also intersects the closed unit disk. Therefore, in any case we may apply Theorem 2.1 to obtain the following fact. 
Corollary 2.2. Let $T$ be a hypercyclic bilateral weighted shift. Then there always exists an infinite-dimensional closed subspace of hypercyclic vectors.

As noted by Salas [Sa2], a characterization similar to conditions (1) above can be given for a bilateral backward weighted shift to be hypercyclic. Of course, it can be proved that a hypercyclic bilateral backward shift also satisfies the Hypercyclicity Criterion for a subsequence of positive integers $\left\{n_{k}\right\}$. These results are not in contradiction with the result of Salas Sa1] in which he constructed a bilateral shift which does not satisfies the Hypercyclicity Criterion for the whole sequence of natural numbers. Finally, as in [Sa2], we may deduce that there is a hypercyclic operator $T$ such that $T$ and its adjoint $T^{\star}$ have an infinite-dimensional closed subspace of hypercyclic vectors.

2. Backward weighted shifts. Let $\left\{e_{n}\right\}_{n>0}$ be the canonical basis of $\ell^{2}$. The backward weighted shift $T: \ell^{2} \rightarrow \ell^{2}$ is defined by $T e_{n}=w_{n} e_{n-1}$ for $n \geq 1$ and $T e_{0}=0$, where the weight sequence $\left\{w_{n}\right\}_{n \geq 1}$ is a bounded sequence of positive numbers. Gethner and Shapiro GS, by means of the Hypercyclicity Criterion, proved that if $\lim \prod_{j=1}^{n} w_{j}=\infty$, then $T$ is hypercyclic. Salas [Sa2] proved that a backward weighted shift is hypercyclic if and only if $\limsup _{n \rightarrow \infty} \prod_{j=1}^{n} w_{j}=\infty$.

In order to apply Theorem 2.1 let us show that a backward weighted shift always satisfies the Hypercyclicity Criterion whenever it is hypercyclic. As before we take $X=Y=\operatorname{span}\left\{e_{n}\right\}$, and the operator $S$ will be the weighted forward shift defined on $\ell^{2}$ by

$$
S e_{n}=\frac{1}{w_{n+1}} e_{n+1} \quad(n=0,1,2, \ldots) .
$$

Clearly, $T S=$ identity on $\ell^{2}$. Since $T$ is hypercyclic we have $\lim \sup _{n \rightarrow \infty} \prod_{j=1}^{n} w_{j}=$ $\infty$. Thus for every $\varepsilon>0$ and for every positive integer $q$ there is an $n_{k}$ such that

$$
\prod_{i=1}^{n_{k}} w_{i+j}>\frac{1}{\varepsilon} \quad(j \leq q)
$$

Again, let $\left\{\varepsilon_{k}\right\}_{k \geq 1}$ be any sequence of positive numbers decreasing to zero. For each positive integer $k$ we can find a positive integer $n_{k}$ such that condition (2) is satisfied for $\varepsilon=\varepsilon_{k}$ and $q=k$. Let $x \in \operatorname{span}\left\{e_{n}\right\}$; then $x=\sum_{j=0}^{k_{0}} \alpha_{j} e_{j}$ and $T^{n_{k}} x$ is eventually zero. As before, for $k \geq k_{0}$ we can prove that $\left\|S^{n_{k}} x\right\|<\varepsilon_{k}\|x\|$. Thus $T$ satisfies the Hypercyclicity Criterion.

In Section 4 (see Proposition 4.1) we will prove that the nearest part of the essential spectrum of a backward weighted shift is a circle centered at the origin and of radius

$$
r_{1}=\lim _{n \rightarrow \infty}\left(\inf _{k} \prod_{i=0}^{n-1} w_{k+i}\right)^{1 / n} .
$$

If $r_{1}=0$, then the circle degenerates to a point. For the existence of the above limit see [Shi, Propositions 12 and 14] or Section 4. Thus the essential spectrum intersects the closed unit disk if and only if $r_{1} \leq 1$. Thus we may apply Theorem 2.1 again to obtain

Corollary 2.3. Let $T$ be a backward weighted shift with positive weight sequence $\left\{w_{n}\right\}$. Then $T$ has an infinite-dimensional closed subspace of hypercyclic vectors if and only if $\lim \sup _{n} \prod_{i=1}^{n} w_{i}=\infty$ and $r_{1} \leq 1$. 
3. The identity plus a backward weighted shift. Let $T$ be a backward weighted shift as above. Chan and Shapiro [CS] proved that for certain types of unilateral backward weighted shifts $T$ on Hilbert space the operator $I+T$ is hypercyclic. Godefroy and Shapiro GoS asked whether $I+T$ is hypercyclic on a Hilbert space if $T$ is a quasinilpotent unilateral backward weighted shift. A similar question was asked also in $\mathrm{CS}$. In $\underline{\mathrm{Sa} 2}$ H. Salas proved that for a backward weighted shift $T: \ell^{2} \rightarrow \ell^{2}$ with positive weights, $I+T$ is hypercyclic. In LM] Proposition 4.3 and remark following it] it is proved (using Salas's method) that the operator $I+T$ always satisfies the Hypercyclicity Criterion. Therefore, a direct application of Theorem 2.1 gives the following

Corollary 2.4. Let $T$ be a backward weighted shift. Then the operator $I+T$ has an infinite-dimensional closed subspace of hypercyclic vectors if and only if $r_{1} \leq 2$.

Corollary 2.4 answers one of the questions posed in [LM].

4. Multiplication operators. Following Godefroy and Shapiro [GoS], let $\Omega \subset \mathbb{C}$ be a region (that is, a connected, open subset of $\mathbb{C}$ ) and let $\mathcal{H}$ be a Hilbert space of holomorphic function on $\Omega$ subject to the restrictions of non-triviality (that is, $\mathcal{H} \neq 0$ ) and bounded point evaluations (for each $z \in \Omega$, the evaluation functional $f \rightarrow f(z)$ is continuous on $\mathcal{H})$. A complex valued function $\varphi$ on $\Omega$ for which the pointwise product $\varphi f \in \mathcal{H}$ for every $f \in \mathcal{H}$ is called a multiplier. Each multiplier of $\mathcal{H}$ determines a linear multiplication operator $M_{\varphi}$ by the formula

$$
M_{\varphi} f=\varphi f \quad(f \in \mathcal{H}) .
$$

The boundedness of $M_{\varphi}$ follows from the point continuity evaluations and the Closed Graph Theorem. Proposition 4.4 in $[\mathrm{GoS}]$ proves that every multiplier is a bounded holomorphic function on $\Omega$. Godefroy and Shapiro GoS, Theorem 4.9] also proved that if

$$
\left\|M_{\varphi}\right\|=\|\varphi\|_{\infty}=\sup \{|\varphi(z)|: z \in \Omega\},
$$

then the adjoint $M_{\varphi}^{\star}$ is hypercyclic if and only if $\varphi(\Omega)$ intersects the unit circle.

From part b) of Proposition 4.4 in [GoS] it can be deduced that the spectrum of $M_{\varphi}^{\star}$ is the closure of $\overline{\varphi(\Omega)}=\{\bar{z}: z \in \varphi(\Omega)\}$. In addition, if $\varphi$ is one-to-one, then the essential spectrum of $M_{\varphi}^{\star}$ is the boundary $\partial \overline{\varphi(\Omega)}$. Therefore, we have the following fact.

Corollary 2.5. Suppose every bounded function $\varphi$ on $\Omega$ is a multiplier of $\mathcal{H}$, with $\left\|M_{\varphi}\right\|=\|\varphi\|_{\infty}$. Then whenever $\varphi$ is one-to-one the operator $M_{\varphi}^{\star}$ has an infinitedimensional closed subspace of hypercyclic vectors if and only if $\varphi(\Omega)$ intersects the unit circle and the boundary $\partial \varphi(\Omega)$ intersects the closed unit disk.

5. Differentiation and translation operators. Following Chan and Shapiro [CS], let us call an entire function $\gamma(z)=\sum \gamma_{n} z^{n}$ a comparison function if $\gamma_{n}>0$ for each $n$, and the sequence of ratios $\gamma_{n+1} / \gamma_{n}$ decreases to zero as $n$ increases to $\infty$. If the sequence $(n+1) \gamma_{n+1} / \gamma_{n}$ is monotonically decreasing to zero, then $\gamma$ is called an admissible comparison function. For each comparison function $\gamma$, it can be defined $E^{2}(\gamma)$ to be the Hilbert space of power series:

$$
f(z)=\sum_{n=0}^{\infty} \hat{f}(n) z^{n}
$$


for which the norm

$$
\|f\|_{2, \gamma}^{2}=\sum_{n=0}^{\infty} \gamma_{n}^{-2}|\hat{f}(n)|^{2}<\infty .
$$

It can be said that the restrictions on the growth of comparison functions are reflected in the behavior of the functions in the corresponding Hilbert space (see [CS, p. 1429]).

Proposition 1.1 in CS states that the differentiation operator $D: E^{2}(\gamma) \rightarrow$ $E^{2}(\gamma)$, which assigns to each function $f \in E^{2}(\gamma)$ its derivative $f^{\prime}$, is bounded on $E^{2}(\gamma)$ if and only if $n \gamma_{n} / \gamma_{n-1}$ is bounded. In fact they proved that it is a backward weighted shift with respect to the orthonormal basis $e_{n}=\gamma_{n} z^{n}$ with positive weight sequence $w_{k}=k \gamma_{k} / \gamma_{k-1}$. So, applying Corollary 2.3, we have the following.

Corollary 2.6. Suppose that $\gamma$ is a comparison function. The operator of differentiation has an infinite-dimensional closed subspace of hypercyclic vectors if and only if

$$
\limsup k ! \gamma_{k}=\infty \quad \text { and } \quad \lim _{n \rightarrow \infty}\left(\inf _{k} \frac{(n+k-1) !}{(k-1) !} \frac{\gamma_{n+k-1}}{\gamma_{k-1}}\right)^{1 / n} \leq 1 .
$$

From the fact that the differentiation operator $D$ is bounded, and the fact that the translation operator by a complex number $a$, which assigns to each function $f(z)$ the function $f(z+a)$, satisfies the equation $T_{a}=e^{a D}$, Chan and Shapiro [CS. Corollary 1.2] proved that the translation operator $T_{a}$ is also bounded whenever $n \gamma_{n} / \gamma_{n-1}$ is bounded.

From the equation $T_{a}=e^{a D}$ we see that the essential spectrum of $T_{a}$ contains a closed curve which passes through $e^{-|a| r_{1}}$ and $e^{|a| r_{1}}$ if $r_{1}>0$ and $\{1\}$ if $r_{1}=0$. In any case, $\sigma_{e}\left(T_{a}\right)$ intersects the closed unit disk. Since the translation operator also satisfies the Hypercyclicity Criterion (see [CS]), we have

Corollary 2.7. If $\gamma$ is a comparison function, then there is an infinite-dimensional Hilbert subspace of hypercyclic vectors for the translation operator $T_{a}$.

This answers another question posed in [LM]. Corollary 2.7 was obtained in [LM] only for admissible comparison functions.

6. Composition operators. We close this section with composition operators, which were the first class of operators to which Theorem A was applied. Let $\mathcal{H}=\mathcal{H}^{2}(\mathbb{D})$ be the Hardy space of analytic functions on the unit disk $\mathbb{D}$ and whose boundary values are in $L^{2}(\partial \mathbb{D})$.

If $\varphi$ is holomorphic on $\mathbb{D}$ and $\varphi(\mathbb{D}) \subset \mathbb{D}$, the Littlewood Subordination Theorem (see [Sh Chapter 1]) asserts that the corresponding composition operator $C_{\varphi}$ which assigns to each function $f \in \mathcal{H}^{2}(\mathbb{D})$ the function $C_{\varphi}(f)=f \circ \varphi$ takes $\mathcal{H}^{2}(\mathbb{D})$ boundedly into itself.

In [BS1], Bourdon and Shapiro gave a complete characterization of those linear fractional transformations which induce hypercyclic operators. In [BS2], they extend their results to a larger class of inducing maps. The second author by means of Theorem A proved that hypercyclic composition operators induced by linear fractional transformations have an infinite-dimensional closed subspace of hypercyclic vectors (see [Mo, Section 3]). Theorem 2.1 clarifies why hypercyclic composition operators were so good in satisfying Theorem A. The fact is that the 
essential spectrum of hypercyclic composition operators induced by linear fractional transformations always intersects the closed unit disk.

Theorem 2.1 also applies to hypercyclic composition operators studied in BS2. In fact, Theorems 4.7 and 4.16 in [BS2] give sufficient conditions for a composition operator $C_{\varphi}$ to be hypercyclic. Under the hypotheses of Theorem 4.7 in BS2 we can apply Lemma 7.24 in [CM] to see that the essential spectrum of $C_{\varphi}$ always intersects the closed unit disk. Also, under the hypotheses of Theorem 4.16 in BS2 we can apply Corollary 7.39 in $[\mathrm{CM}$ to see that the essential spectrum of $C_{\varphi}$ always contains the unit circle. Therefore, applying Theorem 2.1 we find that under the hypotheses of Theorem 4.7 or 4.16 in BS2] there always exists an infinitedimensional closed vector space of hypercyclic vectors.

\section{The ESSENTIAL SPECTRUM INTERSECTS}

In this section we will prove that if the essential spectrum of an operator satisfying the Hypercyclicity Criterion intersects the closed unit disk, then there is an infinite-dimensional closed subspace of hypercyclic vectors.

We begin with a result that asserts that for hypercyclic operators the essential spectrum and the left essential spectrum coincide. This fact will be very useful in this section and in Section 5 .

Proposition 3.1. If $T$ is a hypercyclic bounded operator on a separable Hilbert space $\mathcal{H}$, then $\sigma_{e}(T)=\sigma_{l e}(T)$.

Proof. Since $\sigma_{l e}(T)$ is contained in $\sigma_{e}(T)$, we need only prove the other inclusion. If $\lambda$ does not belong to $\sigma_{l e}(T)$, then $\operatorname{ran}(T-\lambda)$ is closed. Since $T$ is hypercyclic, $\operatorname{ran}(T-\lambda)$ is dense in $\mathcal{H}$ (see [Ki, Theorem 2.3]). Therefore, $\operatorname{ran}(T-\lambda)=\mathcal{H}$ and, consequently, $\lambda$ does not belong to $\sigma_{e}(T)$. The proof is finished.

One of the main keys for the proof of our result in this section is the following proposition, which is a version of the Apostol-Foiaş-Voiculescu Theorem about normal restrictions of compact perturbations of the identity (see [He1, Theorem 3.49]). The proof is the argument g) implies d) in [Co Chapter XI, Theorem 2.5]. We include the proof not only for the sake of completeness, but also because it gives insight in our results.

Proposition 3.2. Given a bounded linear operator $T, \lambda \in \sigma_{l e}(T)$ and $\varepsilon>0$, there exist an infinite-dimensional subspace $\mathcal{H}_{\varepsilon}$ and a compact operator $K_{\varepsilon}$ such that $\left\|K_{\varepsilon}\right\|<\varepsilon,\left(T-K_{\varepsilon}\right) \mathcal{H}_{\varepsilon} \subset \mathcal{H}_{\varepsilon}$, and the restriction of $T-K_{\varepsilon}$ to $\mathcal{H}_{\varepsilon}$ is $\lambda I_{\varepsilon}$, where $I_{\varepsilon}$ is the identity on $\mathcal{H}_{\varepsilon}$.

Proof. Since $\lambda \in \sigma_{l e}(T)$, the operator $T-\lambda$ is not a left Fredholm operator. Therefore, there is an orthonormal system $\left\{e_{n}\right\}$ such that

$$
\lim _{n}\left\|(T-\lambda) e_{n}\right\|=0
$$

(see [Co, Chapter XI, Theorem 2.5]). By extracting a subsequence, if necessary, we may suppose that

We define

$$
\left\|(T-\lambda) e_{n}\right\|<\frac{\varepsilon}{2^{n}} \quad(n \geq 1)
$$

$$
K_{\varepsilon}(x)=\sum_{n=1}^{\infty}\left\langle x, e_{n}\right\rangle(T-\lambda) e_{n}
$$


Since $K_{\varepsilon}$ is the limit of finite rank operators, we find that $K_{\varepsilon}$ is compact. On the other hand, it easy to see that $\left\|K_{\varepsilon}\right\|<\varepsilon$. If we define $\mathcal{H}_{\varepsilon}=\overline{\operatorname{span}}\left\{e_{n}\right\}$, then we have

$$
\left(T-K_{\varepsilon}\right) e_{n}=T e_{n}-T e_{n}+\lambda e_{n}=\lambda e_{n},
$$

and all the required properties are satisfied.

We remark here that in order to prove our results we do not even require the full strength of Proposition 3.2.

Finally, some elementary theory about basic sequences will be required. Recall that $\left\{x_{n}\right\} \subset \mathcal{H}$ is a basic sequence if for each $x$ belonging to the closed linear span of $\left\{x_{n}\right\}$ there exists a unique sequence of scalars $\left\{\alpha_{n}\right\}$ such that

$$
x=\sum_{n=1}^{\infty} \alpha_{k} x_{k} .
$$

For instance, an orthonormal system in a Hilbert space is a basic sequence. The coefficient functionals $\left\{x_{n}^{\star}\right\}$ are defined as the linear functionals $x_{k}^{\star}\left(\sum_{n=1}^{\infty} \alpha_{k} x_{k}\right)=$ $\alpha_{k}$. These functionals are continuous (see [Di, pp. 32-33]) and can be extended by the Hahn-Banach Theorem to $\mathcal{H}$.

Two basic sequences are called equivalent if the convergence of $\sum \alpha_{n} x_{n}$ is equivalent to that of $\sum \alpha_{n} y_{n}$. If $\left\{x_{n}\right\}$ and $\left\{y_{n}\right\}$ are basic sequences, then there is an obvious isomorphism between their closed linear spans, $\overline{\operatorname{span}}\left\{x_{n}\right\}$ and $\overline{\operatorname{span}}\left\{y_{n}\right\}$. We also need the following standard stability theorem for bases (see Di, Theorem 9 , p. 46]).

Theorem 3.3. Let $\left\{y_{n}\right\}$ be a basic sequence in a Hilbert space $\mathcal{H}$ and suppose that $\left\{y_{n}^{\star}\right\}$ is the sequence of coefficient functionals. Suppose that $\left\{z_{n}\right\}$ is a sequence in $\mathcal{H}$ for which $\sum\left\|y_{n}^{\star}\right\|\left\|y_{n}-z_{n}\right\|<1$. Then $\left\{z_{n}\right\}$ is a basic sequence equivalent to $\left\{y_{n}\right\}$.

Now we turn to the proof of the spectral sufficient condition for the existence of an infinite-dimensional closed subspace of hypercyclic vectors. Observe that the Hypercyclicity Criterion makes a new appearance.

Theorem 3.4. Let $T$ be a bounded linear operator on a separable Hilbert space $\mathcal{H}$ satisfying the Hypercyclicity Criterion. Suppose also that the essential spectrum of $T$ intersects the closed unit disk. Then there exists an infinite-dimensional closed subspace of hypercyclic vectors for $T$.

Proof. Since the operator $T$ satisfies the Hypercyclicity Criterion, condition a) of Theorem A is satisfied for some subsequence of positive integers. Therefore, we have only to prove that condition b) of Theorem A is satisfied for the same subsequence.

Suppose the essential spectrum $\sigma_{e}(T)$ intersects the closed unit disk. Since $T$ is hypercyclic, Proposition 3.1 implies that $\sigma_{l e}(T)=\sigma_{e}(T)$. Thus $\sigma_{l e}(T)$ also intersects the closed unit disk. Thus we can take $\lambda \in \sigma_{l e}(T)$ with $|\lambda| \leq 1$. By Proposition 3.2 we can find a compact operator $K$ and an infinite-dimensional Hilbert space $\mathcal{H}^{\prime}$ such that $\mathcal{H}^{\prime}$ is invariant under $T-K$ and the matrix representation for some orthonormal basis is

$$
\left(\begin{array}{cc}
N & * \\
0 & A
\end{array}\right)
$$


where $N$ is an infinite-diagonal matrix with diagonal entries equal to $\lambda$. Here $N$ is the matrix representation of restriction of $T-K$ to $\mathcal{H}^{\prime}$. In fact the norm of $K$ can be taken as small as desired, but this is irrelevant for our purposes. However, observe that the norm of the restriction of $T-K$ to $\mathcal{H}^{\prime}$ is $\leq 1$.

Although the rest of the proof runs parallel to that of Theorem 2.1 in [LM], the details are different. The algebraic properties of compact operators imply that, for each positive integer $n$,

$$
T^{n}=(T-K+K)^{n}=(T-K)^{n}+K_{n},
$$

where $K_{n}$ is a compact operator. Let $\left\{e_{n}\right\}$ be the orthonormal basis of $\mathcal{H}^{\prime}$ for which $T-K$ has the matrix representation (1). We will construct a subsequence $\left\{y_{n}\right\} \subset\left\{e_{n}\right\}$ such that for every $x=\sum_{j=1}^{\infty} \alpha_{j} y_{j} \in \overline{\operatorname{span}}\left\{y_{n}\right\}$ and for every positive integer $n$ the following inequality is satisfied:

$$
\left\|K_{n} \sum_{j=n}^{\infty} \alpha_{j} y_{j}\right\|<\left\|\sum_{j=n}^{\infty} \alpha_{j} y_{j}\right\| .
$$

In order to do this we consider a sequence $\left\{\varepsilon_{m}\right\}$ of positive numbers such that $\sum_{m=1}^{\infty} \varepsilon_{m}^{2}<1$. Since $K_{1}$ is compact and $\left\{e_{n}\right\}$ converges weakly to zero, we have $\left\|K_{1} e_{n}\right\| \rightarrow 0$. Thus we can choose a positive integer $m_{1}$ large enough to have $\left\|K_{1} e_{m}\right\|<\varepsilon_{1}$ for every $m \geq m_{1}$. In this way we can choose $m_{n} \geq m_{n-1}$ such that $\left\|K_{n} e_{m}\right\|<\varepsilon_{m}$ for every $m \geq m_{n}$. We define $y_{n}=e_{m_{n}}$. Therefore, if $\sum_{j=1}^{\infty} \alpha_{j} y_{j} \in$ $\overline{\operatorname{span}}\left\{y_{n}\right\}$ we have, for each positive integer $n$,

$$
\begin{aligned}
\left\|K_{n} \sum_{j=n}^{\infty} \alpha_{j} y_{j}\right\| & \leq \sum_{j=n}^{\infty}\left|\alpha_{j}\right|\left\|K_{n} y_{j}\right\| \\
& <\sum_{j=n}^{\infty}\left|\alpha_{j}\right| \varepsilon_{j} \\
& \leq\left(\sum_{j=n}^{\infty}\left|\alpha_{j}\right|^{2}\right)^{\frac{1}{2}}\left(\sum_{j=n}^{\infty} \varepsilon_{j}^{2}\right)^{\frac{1}{2}} \\
& <\left\|\sum_{j=n}^{\infty} \alpha_{j} y_{j}\right\| .
\end{aligned}
$$

It is important to observe that we are free to choose the sequence $\left\{m_{n}\right\}$ contained in the sequence for which the Hypercyclicity Criterion is satisfied.

Let $X \subset \mathcal{H}$ be the dense subset required by the Hypercyclicity Criterion. We choose a sequence $\left\{z_{n}\right\} \subset X$ such that for each positive integer $n$

$$
\left\|y_{n}-z_{n}\right\|<\frac{\varepsilon_{n}}{\max \left\{\|T\|, \ldots,\left\|T^{n}\right\|\right\}} .
$$

Since $T$ is hypercyclic, $\|T\|>1$ and also $\left\|y_{n}-z_{n}\right\|<\varepsilon_{n}$ for every $n$. In addition, we may suppose that the sequence $\left\{\varepsilon_{n}\right\}$ also satisfies $\sum_{n=1}^{\infty} \varepsilon_{n}<1$. Let $\left\{y_{n}^{\star}\right\}_{n \geq 1}$ be the sequence of coefficient functionals corresponding to the orthonormal sequence 
$\left\{y_{n}\right\}$. Thus

$$
\sum_{n=1}^{\infty}\left\|y_{n}^{\star}\right\|\left\|y_{n}-z_{n}\right\|<\sum_{n=1}^{\infty} \varepsilon_{n}<1 .
$$

Therefore, upon applying Theorem 3.3 we find that every subsequence $\left\{z_{n_{k}}\right\}$ is a basic sequence equivalent to the corresponding orthonormal sequence $\left\{y_{n_{k}}\right\}$. We are in position to construct a basic subsequence of $\left\{z_{n}\right\}$ whose closed linear span will be the space $\mathcal{H}_{0}$ we are looking for. Set $n_{1}=1$. Since $z_{n_{1}} \in X$, we may apply hypothesis a1) of Theorem $\mathrm{A}$ to choose a positive integer $n_{2}>n_{1}$ such that $\left\|T^{n_{2}} z_{n_{1}}\right\| \leq \frac{\varepsilon_{n_{1}}}{2}$. Again, we may choose a positive integer $n_{3}>n_{2}$ large enough to have

$$
\left\|T^{n_{3}} z_{n_{k}}\right\|<\frac{\varepsilon_{n_{k}}}{2^{2}} \quad \text { for } k=1,2 .
$$

In this way we construct an increasing sequence of positive integers $\left\{n_{k}\right\}$ such that

$$
\left\|T^{n_{k}} z_{n_{j}}\right\|<\frac{\varepsilon_{n_{j}}}{2^{k}} \quad \text { for } j=1, \ldots, k-1 .
$$

We define $\mathcal{H}_{0}=\overline{\operatorname{span}}\left\{z_{n_{k}}\right\}$. Let $z=\sum_{j=1}^{\infty} \alpha_{j} z_{n_{j}} \in \mathcal{H}_{0}$. Since $\left\{z_{n_{k}}\right\}$ is a perturbation of an orthonormal system, it is bounded away from zero, so we may assume $\left|\alpha_{j}\right|<\|z\|$. Taking into account the fact that the norm of the restriction of $T-K$ to $\mathcal{H}^{\prime}$ is $\leq 1$ and the fact that $\left\|K_{n_{k}} x_{k}\right\|<\left\|x_{k}\right\|$ for $x_{k}=\sum_{j=k}^{\infty} \alpha_{j} y_{n_{j}}$, and using the inequalities (2), we estimate

$$
\begin{aligned}
\left\|T^{n_{k}} \sum_{j=k}^{\infty} \alpha_{j} z_{n_{j}}\right\| & \leq\left\|\sum_{j=k}^{\infty} \alpha_{j} T^{n_{k}}\left(z_{n_{j}}-y_{n_{j}}\right)\right\|+\left\|T^{n_{k}} \sum_{j=k}^{\infty} \alpha_{j} y_{n_{j}}\right\| \\
& <\sum_{j=k}^{\infty}\left|\alpha_{j}\right|\left\|T^{n_{k}}\right\|\left\|z_{n_{j}}-y_{n_{j}}\right\|+\left\|T^{n_{k}} \sum_{j=k}^{\infty} \alpha_{j} y_{n_{j}}\right\| \\
& <\|z\| \sum_{j=k}^{\infty} \varepsilon_{n_{j}}+\left\|\left(T-K_{n_{k}}\right)^{n_{k}} \sum_{j=k}^{\infty} \alpha_{j} y_{n_{j}}\right\|+\left\|K_{n_{k}} \sum_{j=k}^{\infty} \alpha_{j} y_{n_{j}}\right\| \\
& <\|z\| \sum_{j=k}^{\infty} \varepsilon_{n_{j}}+2\left\|\sum_{j=k}^{\infty} \alpha_{j} y_{n_{j}}\right\| .
\end{aligned}
$$

Finally, using the above estimation in the second inequality below,

$$
\begin{aligned}
\left\|T^{n_{k}} z\right\| & \leq \sum_{j=1}^{k-1}\left|\alpha_{j}\right|\left\|T^{n_{k}} z_{n_{j}}\right\|+\left\|T^{n_{k}} \sum_{j=k}^{\infty} \alpha_{j} z_{n_{j}}\right\| \\
& <\sum_{j=1}^{k-1}\left|\alpha_{j}\right| \frac{\varepsilon_{j}}{2^{k}}+\|z\| \sum_{j=k}^{\infty} \varepsilon_{n_{j}}+2\left\|\sum_{j=k}^{\infty} \alpha_{j} y_{n_{j}}\right\| \\
& <\|z\|\left(\frac{1}{2^{k}}+\sum_{j=k}^{\infty} \varepsilon_{n_{j}}\right)+2\left\|\sum_{j=k}^{\infty} \alpha_{j} y_{n_{j}}\right\| .
\end{aligned}
$$

Upon letting $k$ tend to $\infty$ we have that (3) tends to zero because each of the series which appear in (3) is the remainder of a convergent series. Therefore, we 
have proved that the hypotheses of Theorem A are satisfied for the sequence $\left\{n_{k}\right\}$. Consequently, if $T$ satisfies the Hypercyclicity Criterion and the essential spectrum intersects the closed unit disk, then there is an infinite-dimensional closed subspace of hypercyclic vectors, and the proof is complete.

Remark. From (3) above we have

$$
\left\|T^{n_{k}} z\right\| \leq\|z\|\left(\frac{1}{2^{k}}+\sum_{j=k}^{\infty} \varepsilon_{n_{j}}\right)+2\left\|\sum_{j=k}^{\infty} \alpha_{j} y_{n_{j}}\right\| \leq 4\|z\|
$$

for every positive integer $k$ and for every $z \in \mathcal{H}_{0}$. Thus, under the hypotheses of Theorem 3.4 condition iii) of Theorem 2.1 is satisfied.

\section{The essential minimum modulus}

In this expository section we will define the essential minimum modulus and discuss several of its properties which are relevant to the proof of the converse of Theorem 3.4 in the following section. If $T \in \mathcal{L}(\mathcal{H})$, then the minimum modulus is defined as

$$
m(T)=\min \left\{\lambda \in \sigma\left(T^{\star} T\right)^{\frac{1}{2}}\right\}
$$

and the essential minimum modulus is defined as

$$
m_{e}(T)=\min \left\{\lambda \in \sigma_{e}\left(T^{\star} T\right)^{\frac{1}{2}}\right\} .
$$

This is one of the most standard ways of defining the essential minimum modulus on Hilbert space (see [He1, p. 167]). The essential minimum modulus has been important in understanding the structure of the Calkin algebra, and has also been used to obtain conclusions about the algebra of operators $\mathcal{L}(\mathcal{H})$. For instance, in [Bo, Theorem 3], it is proved that the distance from an operator $T$ to the set of all invertible operators is $\max \left\{m_{e}(T), m_{e}\left(T^{\star}\right)\right\}$ whenever ind $T \neq 0$. Theorem 4 in [Bo] shows that the distance from an operator $T$ to the set of Fredholm operators is $\max \left\{m_{e}(T), m_{e}\left(T^{\star}\right)\right\}$ whenever $T$ is not Fredholm.

First we will see several properties of the minimum modulus that have their corresponding counterparts in the essential minimum modulus. By using the polar factorization of $T$, it is easy to check that (see $[\mathrm{BO}$, Theorem 1, (i)])

$$
m(T)=\inf \{\|T x\|:\|x\|=1\} .
$$

The minimum modulus has a nice geometric interpretation. Let $\mathcal{M}(\mathcal{H})$ denote the set of operators $T$ such that $\operatorname{ker} T=\{0\}$ and $\operatorname{ran} T$ is closed. Clearly, $\mathcal{M}(\mathcal{H})$ is open in $\mathcal{L}(\mathcal{H})$. It is well-known that $m(T)>0$ if and only if $T$ belongs to $\mathcal{M}(\mathcal{H})$ (see [Bo, Theorem 1, (ii)]). Proposition 2 in [MZ shows that

$$
m(T)=\operatorname{dist}(T, \mathcal{L}(\mathcal{H}) \backslash \mathcal{M}(\mathcal{H})) .
$$

Furthermore, denoting by $b(T)$ the supremum of all $\varepsilon \geq 0$ such that $m(T-\lambda I)>0$ for $|\lambda|<\varepsilon$, we have the following asymptotic formula (see [MZ, Theorem 3]):

$$
b(T)=\lim _{n \rightarrow \infty} m\left(T^{n}\right)^{1 / n} .
$$

For instance, if $T^{\star}$ is the adjoint of a backward weighted shift $T$ with positive weight sequence $\left\{w_{n}\right\}$, then

$$
b\left(T^{\star}\right)=r_{1}\left(T^{\star}\right),
$$


where $r_{1}\left(T^{\star}\right)=r_{1}$ is defined as in formula (3) in Section 2 (see Shi, Propositions 12 and 14]). As an application of formula (3) above we will prove the following proposition, which completes the proof of Corollary 2.3 in Section 2.

Proposition 4.1. If $T$ is a backward weighted shift, then the nearest part to the origin of the essential spectrum is the circle centered at the origin of radius $r_{1}\left(T^{\star}\right)$.

Proof. First, observe that for any complex number $\lambda$ the operator $T^{\star}-\lambda$ is injective. Hence, by the definition of $b\left(T^{\star}\right)$ we have that for any $\varepsilon>0$ we can find $r_{1}\left(T^{\star}\right) \leq$ $\left|\lambda_{1}\right|<r_{1}(T)+\varepsilon$ such that $T^{\star}-\lambda_{1}$ is not closed. Therefore, $\lambda_{1}$ belongs to the essential spectrum of $T^{\star}$. Since $\sigma_{e}\left(T^{\star}\right)$ is compact, we find that there is, at least, one point on the circle $|z|=r_{1}\left(T^{\star}\right)$ that belongs to $\sigma_{e}\left(T^{\star}\right)$. By circular symmetry (see [Shi, Corollary 2, p. 52]) the whole circle $|z|=r_{1}\left(T^{\star}\right)$ must be contained in the essential spectrum.

Second, let us prove that if $|\lambda|<r_{1}\left(T^{\star}\right)$, then $\lambda$ does not belong to $\sigma_{e}\left(T^{\star}\right)$. Toward this end, we consider the quantity

$$
r_{2}\left(T^{\star}\right)=\liminf _{n \rightarrow \infty}\left(\prod_{i=1}^{n} w_{i}\right)^{1 / n} .
$$

Observe that $r_{1}\left(T^{\star}\right) \leq r_{2}\left(T^{\star}\right)$. If $|\lambda|<r_{1}\left(T^{\star}\right) \leq r_{2}\left(T^{\star}\right)$, then $\operatorname{dim} \operatorname{ker}\left(T^{\star}-\lambda\right)^{\star}=$ $\operatorname{dim} \operatorname{ker}(T-\bar{\lambda})=1$ (see [Shi, Theorem 8, p. 70]). Also $\operatorname{ker}\left(T^{\star}-\lambda\right)=\{0\}$, and by the definition of $b\left(T^{\star}\right)$ we have that the range of $T^{\star}-\lambda$ is closed. Upon putting everything together we see that $\lambda$ is not in the essential spectrum of $T^{\star}$.

Finally, the result follows from the fact that $\sigma_{e}(T)=\left\{\bar{\lambda}: \lambda \in \sigma_{e}\left(T^{\star}\right)\right\}$.

The rest of this section is based on the work of Bouldin [Bo] and Zemánek [Ze1]. Let us prove that the essential minimum modulus satisfies a formula analogous to (1). Recall that $\pi(T)=\tilde{T}$ denotes the projection of $T$ onto the Calkin algebra. The formula $\pi\left(T^{\star}\right)=(\pi(T))^{\star}$ can be used to define an involution which makes the Calkin algebra into a $C^{\star}$-algebra, and the projection $\pi$ from $\mathcal{L}(\mathcal{H})$ onto the Calkin algebra is a $\star$-homomorphism. It is not difficult to check that

$$
\pi\left(\left(T^{\star} T\right)^{1 / 2}\right)=\left(\pi\left(T^{\star}\right) \pi(T)\right)^{1 / 2} .
$$

Now, the elements of any $C^{\star}$-algebra can be regarded as operators on a Hilbert space $\mathcal{H}_{1}$ with norm $|$.$| and such that |\tilde{T}|=\|\tilde{T}\|_{e}$, where $\|\tilde{T}\|_{e}$ denotes the norm of $\tilde{T}$ in the Calkin algebra (see [Co, Chapter VIII, Theorem 5.17]), for instance). Thus the notation $m(\tilde{T})$ makes sense, and it follows from (1) and (4) that

$$
m_{e}(T)=m(\tilde{T})=\inf \left\{|\tilde{T} x|: x \in \mathcal{H}_{1},|x|=1\right\} .
$$

Now we turn to the geometric interpretation of the essential minimum modulus. Let $\mathcal{S F}_{+}(\mathcal{H})$ denote the set of all operators such that $\operatorname{ran} T$ is closed and $\operatorname{ker} T$ is finite dimensional. It is well-known and not difficult to show that $\mathcal{S} \mathcal{F}_{+}(\mathcal{H})$ is open in $\mathcal{L}(\mathcal{H})$. The following theorem, due to Zemánek (see [Ze1 p. 226]), provides a geometric interpretation of the essential minimum modulus analogous to that of $m(T)$.

Theorem 4.2. Let $T$ be a bounded operator on a Hilbert space $\mathcal{H}$. Then the essential minimum modulus $m_{e}(T)$ is equal to the distance from the operator $T$ to the closed set $\mathcal{L}(\mathcal{H}) \backslash \mathcal{S} \mathcal{F}_{+}(\mathcal{H})$. 
Proof. By Theorem 2 (iv) in [Bo], $m_{e}(T)>0$ if and only if $T$ belongs to $\mathcal{S F}_{+}(\mathcal{H})$. Therefore, to show that $m_{e}(T) \geq \operatorname{dist}\left(T, \mathcal{L}(\mathcal{H}) \backslash \mathcal{S F}_{+}(\mathcal{H})\right)$ it is enough to prove that $m_{e}(S)>0$ whenever $\|T-S\|<m_{e}(T)$. By using the expression (5) above for the essential minimum modulus and the fact that $|\tilde{T}|=\|\tilde{T}\|_{e} \leq\|T\|$ we have, for each $x \in \mathcal{H}_{1}$ with $|x|=1$,

$$
|\tilde{S} x| \geq|\tilde{T} x|-|(\tilde{T}-\tilde{S}) x| \geq m_{e}(T)-|\tilde{T}-\tilde{S}| \geq m_{e}(T)-\|T-S\|>0 .
$$

Upon taking the infimum over all $x \in \mathcal{H}_{1}$ with $|x|=1$, we see that $m_{e}(S)>0$.

Now, given $\varepsilon>0$, we must find an operator $S$ in $\mathcal{L}(\mathcal{H}) \backslash \mathcal{S} \mathcal{F}_{+}(\mathcal{H})$ such that

$$
\|T-S\| \leq m_{e}(T)+\varepsilon .
$$

Toward this end, let us consider the spectral resolution $E\left[\right.$.] of the operator $\left(T^{\star} T\right)^{1 / 2}$. By Theorem 2 (ii) in [Bo the subspace $E\left[m_{e}(T), m_{e}(T)+\varepsilon\right] \mathcal{H}$ has infinite dimension, and since $E\left[m_{e}(T), m_{e}(T)+\varepsilon\right] \leq E\left[0, m_{e}(T)+\varepsilon\right]$ the subspace $\mathcal{H}^{\prime}=$ $E\left[0, m_{e}(T)+\varepsilon\right] \mathcal{H}$ is also infinite-dimensional. Let us define the operator $S$ by $S y=0$ when $y \in \mathcal{H}^{\prime}$, and by $S z=T z$ when $z$ is in the orthogonal complement of $\mathcal{H}^{\prime}$. Clearly, the operator $S$ is in $\mathcal{L}(\mathcal{H}) \backslash \mathcal{S} \mathcal{F}_{+}(\mathcal{H})$. Let $x$ be an arbitrary vector of norm one in $\mathcal{H}$. Set $x=y+z$, where $y \in \mathcal{H}^{\prime}$ and $z$ is in the orthogonal complement of $\mathcal{H}^{\prime}$. Then we have $(T-S) x=T y$. Therefore, $\|(T-S) x\|=\|T y\| \leq m_{e}(T)+\varepsilon$. For a proof of the last inequality see [Co Chapter XI, Theorem 2.5, e) implies f)]. The proof is completed.

The preceding theorem was used by Zemánek (see [Ze1]) to define the essential minimum modulus in the Banach space setting.

Now, Theorem 4.2 implies that if $|\lambda|<m_{e}(T)$, then $T-\lambda$ is in $\mathcal{S F}_{+}(\mathcal{H})$. Thus $\lambda$ is not in $\sigma_{l e}(T)$. Hence, $m_{e}(T) \leq \operatorname{dist}\left(0, \sigma_{l e}(T)\right)$. Although sometimes there is equality in the last inequality (see [AFHV, p. 159]), there are operators for which $m_{e}(T)<\operatorname{dist}\left(0, \sigma_{l e}(T)\right)$. In fact, if $T$ is a unilateral forward shift, $T-\lambda$ is injective for any complex number $\lambda$. Thus $m_{e}(T)=m(T)$. Using Proposition 4.1 and the fact that $m(T)$ can be explicitly computed in terms of the weights (see [Shi. Proposition 14, p. 68]), it is not difficult to construct a unilateral forward weighted shift for which $m_{e}(T)<\operatorname{dist}\left(0, \sigma_{l e}(T)\right)$. However, the following deep theorem of Zemánek (see [Ze2, Theorem 1]) asserts that it is still true that dist $\left(0, \sigma_{l e}(T)\right)$ can be expressed as an asymptotic formula involving $m_{e}\left(T^{n}\right)$.

Theorem 4.3. If $T$ is a bounded linear operator on a separable Hilbert space, then

$$
\lim _{n \rightarrow \infty} m_{e}\left(T^{n}\right)^{1 / n}=\operatorname{dist}\left(0, \sigma_{l e}(T)\right) .
$$

The above theorem will play a critical role in the proof of the converse of Theorem 3.4 .

We close this section by stating another property of $m_{e}(T)$ which will be very useful in the following section (see [He1, Proposition 6.10, ii), p. 167]).

Proposition 4.4. Let $T$ be a bounded linear operator on a separable Hilbert space H. Then

$$
m_{e}(T) \leq \liminf _{n}\left\|T e_{n}\right\|
$$

for any orthonormal system $\left\{e_{n}\right\}$, and there is an orthonormal system for which there is equality. 


\section{The ESSEnTial SPECTRUM MUST INTERSECT}

In this section we will prove the converse of Theorem 3.4. This time we do not need the Hypercyclicity Criterion. The proof depends strongly on the properties of the essential minimum modulus. As in the proof of Theorem 3.4 in [Mo], we will use the sliding hump method.

Theorem 5.1. Suppose that $T$ is a hypercyclic bounded operator on a separable Hilbert space $\mathcal{H}$. If the essential spectrum does not intersect the closed unit disk, then every closed subspace of hypercyclic vectors is finite dimensional.

Proof. Let us suppose that there is an infinite-dimensional closed subspace $\mathcal{H}_{1}$ consisting of hypercyclic vectors. We will construct a vector $x \in \mathcal{H}_{1}$ such that

$$
\lim _{k \rightarrow \infty}\left\|T^{k} x\right\|=\infty,
$$

so $x$ cannot be hypercyclic, a contradiction. Since $T$ is hypercyclic, we can apply Proposition 3.1 to see that $\sigma_{e}(T)=\sigma_{l e}(T)$. On the other hand, the fact that $\sigma_{e}(T)$ does not intersect the closed unit disk implies that $\operatorname{dist}\left(0, \sigma_{l e}(T)\right)>1$. Therefore, upon applying Theorem 4.3 in the preceding section we have

$$
\lim _{n \rightarrow \infty}\left(m_{e}\left(T^{n}\right)\right)^{\frac{1}{n}}=\operatorname{dist}\left(0, \sigma_{l e}(T)\right)>1 .
$$

This implies that there exist $\lambda>1$ and a positive integer $k_{0}$ such that

$$
m_{e}\left(T^{n}\right)>\lambda^{n} \quad \text { for each } n \geq k_{0} .
$$

Now, Proposition 4.4 implies that if $\left\{e_{m}\right\}$ is any orthonormal system, then inequality (1) applied to $T^{n}$ yields

$$
\liminf _{m}\left\|T^{n} e_{m}\right\| \geq m_{e}\left(T^{n}\right) .
$$

Therefore, if $\left\{e_{m}\right\}$ is an orthonormal basis of $\mathcal{H}_{1}$, then inequalities (1) and (2) imply that

$$
\liminf _{m}\left\|T^{n} e_{m}\right\| \geq m_{e}\left(T^{n}\right)>\lambda^{n} \quad \text { for each } n \geq k_{0} .
$$

Let $\left\{e_{i}^{\prime}\right\}$ be an orthonormal basis of $\mathcal{H}$. For each element $e_{m_{l}}$ of $\left\{e_{m}\right\}$ we write $T^{k} e_{m_{l}}=\sum_{i=1}^{\infty} a_{k, l, i} e_{i}^{\prime}$, the expansion with respect to $\left\{e_{i}^{\prime}\right\}$. We claim that it is possible to choose a strictly increasing sequence of non-negative integers $\left\{n_{k}\right\}_{k \geq k_{0}}$ and a subsequence $\left\{e_{m_{k}}\right\}_{k \geq k_{0}}$ such that, for every $k \geq k_{0}$,

$$
\begin{gathered}
\left\|T^{k} e_{m_{k}}\right\|>\lambda^{k}, \\
\left\|\sum_{i=n_{k}+1}^{\infty} a_{k, k, i} e_{i}^{\prime}\right\|<\frac{1}{2}, \\
\left\|\sum_{i=1}^{n_{k-1}-1} a_{k, k, i} e_{i}^{\prime}\right\|<\frac{1}{2},
\end{gathered}
$$




$$
\left\|\sum_{i=n_{k}}^{\infty} a_{k+1, l, i} e_{i}^{\prime}\right\|<\frac{1}{k+1}, \quad \text { for } \quad\left(k_{0} \leq l \leq k\right)
$$

$$
\left\|\sum_{i=1}^{n_{j}} a_{j, k, i} e_{i}^{\prime}\right\|<\frac{1}{k} \quad \text { for } \quad\left(k_{0} \leq j<k\right) .
$$

Using (3) for $n=k_{0}$, it is possible to choose $m_{k_{0}}$ large enough to have inequality (4) for $k=k_{0}$. Since $T^{k_{0}} e_{m_{k_{0}}}$ and $T^{k_{0}+1} e_{m_{k_{0}}}$ are already defined, we can choose $n_{k_{0}}$ large enough to have inequalities (5) and (7) for $k=k_{0}$. If we set $n_{k_{0}-1}=1$, then inequality (6) is trivially satisfied for $k=k_{0}$ if we define the sum on the left hand side of (6) as zero. Now suppose that $n_{k_{0}}, \ldots, n_{k-1}$ and $e_{m_{k_{0}}}, \ldots, e_{m_{k-1}}$ satisfying (4) through (8) are already chosen. Since $\left\{e_{m}\right\}$ tends weakly to zero, so do $T^{j} e_{m}$ $\left(k_{0} \leq j \leq k\right)$. This along with (3) allows us to choose $e_{m_{k}}$ to have (4), (6) and (8). Finally, since $T^{k+1} e_{m_{l}}$ is already defined for $k_{0} \leq l \leq k$, we can choose $n_{k}$ large enough such that (5) and (7) are satisfied.

Now, from (4), (5), (6) and the reverse triangle inequality we have

$$
\begin{aligned}
\left\|\sum_{i=n_{k-1}}^{n_{k}} a_{k, k, i} e_{i}^{\prime}\right\| & =\left\|T^{k} e_{m_{k}}-\sum_{i=1}^{n_{k-1}-1} a_{k, k, i} e_{i}^{\prime}-\sum_{i=n_{k}+1}^{\infty} a_{k, k, i} e_{i}^{\prime}\right\| \\
& \geq\left\|T^{k} e_{m_{k}}\right\|-\left\|\sum_{i=1}^{n_{k-1}-1} a_{k, k, i} e_{i}^{\prime}\right\|-\left\|\sum_{i=n_{k}+1}^{\infty} a_{k, k, i} e_{i}^{\prime}\right\| \\
& >\lambda^{k}-1 .
\end{aligned}
$$

Using the orthogonality of the basis $\left\{e_{i}^{\prime}\right\},(7)$ for $l<k$ and (8) for $l>k$, we have for $l \neq k$

$$
\begin{aligned}
\left\|\sum_{l \neq k} \frac{1}{l} \sum_{i=n_{k-1}}^{n_{k}} a_{k, l, i} e_{i}^{\prime}\right\| & \leq \sum_{l \neq k} \frac{1}{l}\left\|\sum_{i=n_{k-1}}^{n_{k}} a_{k, l, i} e_{i}^{\prime}\right\| \\
& =\sum_{l<k} \frac{1}{l}\left\|\sum_{i=n_{k-1}}^{n_{k}} a_{k, l, i} e_{i}^{\prime}\right\|+\sum_{l>k} \frac{1}{l}\left\|\sum_{i=n_{k-1}}^{n_{k}} a_{k, l, i} e_{i}^{\prime}\right\| \\
& \leq \sum_{l<k} \frac{1}{l}\left\|\sum_{i=n_{k-1}}^{\infty} a_{k, l, i} e_{i}^{\prime}\right\|+\sum_{l>k} \frac{1}{l}\left\|\sum_{i=1}^{n_{k}} a_{k, l, i} e_{i}^{\prime}\right\| \\
& <\sum_{l \neq k} \frac{1}{l^{2}} \\
& <\frac{\pi^{2}}{6} .
\end{aligned}
$$


We define $x=\sum_{l=k_{0}}^{\infty} \frac{1}{l} e_{m_{l}}$, which clearly is in $\mathcal{H}_{1}$. Now, for each positive integer $k \geq k_{0}$

$$
\begin{aligned}
\left\|T^{k} x\right\| & =\left\|\sum_{l=1}^{\infty} \frac{1}{l} T^{k} e_{m_{l}}\right\| \\
& =\left\|\sum_{l=1}^{\infty} \frac{1}{l} \sum_{i=1}^{\infty} a_{k, l, i} e_{i}^{\prime}\right\| \\
& \left.=\| \sum_{l=1}^{\infty} \frac{1}{l} \sum_{i=1}^{n_{k-1}-1} a_{k, l, i} e_{i}^{\prime}+\sum_{i=n_{k-1}}^{n_{k}} a_{k, l, i} e_{i}^{\prime}+\sum_{i=n_{k}+1}^{\infty} a_{k, l, i} e_{i}^{\prime}\right) \| \\
& \geq\left\|\sum_{l=1}^{\infty} \frac{1}{l} \sum_{i=n_{k-1}}^{n_{k}} a_{k, l, i} e_{i}^{\prime}\right\| \\
& \geq\left\|\frac{1}{k} \sum_{i=n_{k-1}}^{n_{k}} a_{k, k, i} e_{i}^{\prime}\right\|-\left\|\sum_{l \neq k} \frac{1}{l} \sum_{i=n_{k-1}}^{n_{k}} a_{k, l, i} e_{i}^{\prime}\right\| \\
& >\frac{\lambda^{k}}{k}-\frac{1}{k}-\frac{\pi^{2}}{6} .
\end{aligned}
$$

We have applied the orthogonality of the basis $\left\{e_{i}^{\prime}\right\}$, the reverse triangle inequality and the inequalities (9) and (10). Therefore, $\left\|T^{k} x\right\| \rightarrow \infty$ as $k \rightarrow \infty$. So $x$ cannot be hypercyclic, and we are finished.

Remark. The same arguments of the proof of Theorem 5.1 show that if ii) or iii) in Theorem 2.1 holds, then the essential spectrum intersects the closed unit disk. For if not, exactly as in the proof of Theorem 5.1 we can construct a vector $x$ in any infinite-dimensional closed subspace such that $\lim _{n}\left\|T^{n} x\right\| \rightarrow \infty$. Therefore, so does $\left\|T^{n_{k}} x\right\|$ for every subsequence $\left\{n_{k}\right\}$, in contradiction with ii) or iii).

Proof of Theorem 2.1. Suppose that i) is satisfied, Theorem 5.1 implies that the essential spectrum intersects the closed unit disk. The proof of Theorem 3.4 shows that ii) is satisfied, and the remark following Theorem 3.4 shows that iii) is also satisfied. If ii) is true, then by the remark above the essential spectrum intersects the closed unit disk, and Theorem 3.4 gives i). It should be observed that since we do not know that condition i) is satisfied for the subsequence in the hypothesis of the Hypercyclicity Criterion, we cannot apply Theorem A directly to see that ii) implies i). So we had to take a short detour. The same reasoning gives that iii) implies i). So i) through iii) are equivalent. Theorems 3.4 and 5.1 show the equivalence of i) through iv).

Let us denote by $H C(\mathcal{H})$ the class of all bounded operators which are hypercyclic and by $H C_{\infty}(\mathcal{H})$ the subclass of operators in $H C(\mathcal{H})$ which have an infinitedimensional closed subspace of hypercyclic vectors. Herrero $\mathrm{He} 3$ characterized the norm-closure of $H C(\mathcal{H})$. We will characterize the norm-closure of $H C_{\infty}(\mathcal{H})$. We denote by $\sigma_{0}(T)$ the set of all normal eigenvalues of $T$, that is, the set of isolated points of $\sigma(T)$ which are not in $\sigma_{e}(T)$. Recall that the Weyl spectrum of a bounded operator is defined by

$$
\sigma_{W}(T)=\bigcap\{\sigma(T+K): K \text { is compact }\}
$$


(see [He1, p. 82]). Finally, $\rho_{s-F}(T)=\{\lambda \in \mathbb{C}: T-\lambda$ is semi-Fredholm $\}$. Recall that an operator is called semi-Fredholm if $\operatorname{ran} T$ is closed and either $\operatorname{dim}(\operatorname{ker} T)$ is finite or $\operatorname{dim}\left(\operatorname{ker} T^{\star}\right)$ is finite (see [He1, pp. 10-11]).

Theorem 5.2. A bounded linear operator $T$ is in the closure of $H C_{\infty}(\mathcal{H})$ if and only if $T$ satisfies the conditions

(i) $\sigma_{W}(T) \cup \partial \mathbb{D}$ is connected,

(ii) $\sigma_{0}(T)=\emptyset$,

(iii) ind $(T-\lambda) \geq 0$ for each $\lambda \in \rho_{s-F}(T)$, and

(iv) $\sigma_{e}(T) \cap \overline{\mathbb{D}} \neq \emptyset$.

Proof. First observe that the conditions (i) through (iii) are the conditions that characterize the norm-closure of $H C(\mathcal{H})$ (see [He3] Theorem 2.1]). Theorem 2.1 in $\left[\mathrm{He} 3\right.$ along with Theorem 5.1 implies that if $T$ is in $\mathcal{H} C_{\infty}(\mathcal{H})$, then conditions (i) through (iv) are satisfied. Since the class of all operators satisfying (i)-(iv) is obviously closed, we find that the operators in the closure of $H C_{\infty}(\mathcal{H})$ also satisfy (i)-(iv). On the other hand, suppose that $T$ satisfies conditions (i)-(iv). Then, given $\varepsilon>0$, there exists a compact operator $K_{\varepsilon}$, with $\left\|K_{\varepsilon}\right\|<\varepsilon$, such that $T-K_{\varepsilon}$ satisfies the Hypercyclicity Criterion (see the proof of Theorem 2.1 in [HW]). As the essential spectrum is invariant under compact perturbations, $\sigma_{e}\left(T-K_{\varepsilon}\right)$ also intersects the closed unit disk. Therefore, we can apply Theorem 3.4 to obtain that $T-K_{\varepsilon}$ has an infinite-dimensional closed subspace of hypercyclic vectors. Since $\varepsilon$ was arbitrary, $T$ is in the closure of $H C_{\infty}(\mathcal{H})$. The proof is finished.

We could also have proved Theorem 5.2 following the lines of the proof of theorem 2.1 in $\mathrm{He} 3$. In such a case, we would have obtained that

$$
H C_{\infty}(\mathcal{H})^{-}+\mathcal{K}(\mathcal{H})=\{T \in \mathcal{L}(\mathcal{H}): T \text { satisfies (i), (iii) and (iv) }\}
$$

is a closed subset of $\mathcal{L}(\mathcal{H})$.

By replacing in Theorem 5.2 the condition $\sigma_{e}(T) \cap \overline{\mathbb{D}} \neq \emptyset$ by $\sigma_{e}(T) \cap \mathbb{D}=\emptyset$ and using the methods of Theorem 2.1 in [He3] we may obtain the norm-closure of the class of all hypercyclic operators such that each subspace of hypercyclic vectors is finite dimensional, that is, the norm closure of $H C(\mathcal{H}) \backslash H C_{\infty}(\mathcal{H})$. Finally, observe that if $T \in H C(\mathcal{H}) \backslash H C_{\infty}(\mathcal{H})$, then $\sigma(T)$ has a unique connected component that contains the closed unit disk. For if not, it is easily seem that $\partial \sigma(T)$ (which is contained in $\sigma_{e}(T)$ because $\sigma_{0}(T)=\emptyset$ for hypercyclic operators) intersects the closed unit disk, a contradiction.

\section{Concluding Remark}

It would be interesting to replace the Hypercyclicity Criterion in Theorem 2.1 by the weaker hypothesis that the operator $T$ is hypercyclic. However, it may happen that these two hypotheses are equivalent. In fact, as far as we know all hypercyclic operators that have appeared in the literature satisfies the Hypercyclicity Criterion. Thus the following question remains open

Question. Does every hypercyclic operator satisfy the Hypercyclicity Criterion?

To appreciate the importance of an affirmative answer to this question, observe that if an operator $T$ satisfies the Hypercyclicity Criterion, so do $T^{n}$ and $T \oplus T$. This provides a further proof of a recent result of Ansari $\mathrm{An}$ and a solution to a question posed by Herrero. 


\section{REFERENCES}

[An] S. I. Ansari, Hypercyclic and cyclic vectors, J. Funct. Anal. 128 (1995), 374-383. MR 96h:47002

[AFHV] C. Apostol, L. A. Fialkow, D. A. Herrero and D. Voiculescu, Approximation of Hilbert space operators, Vol. II, Pitman Res. Notes Math., vol. 102, Longman Scientific and Technical, 1984. MR 85m:47002

[Be] B. Beauzamy, Un opérateur sur l'espace de Hilbert, dont tous les polynômes sont hypercycliques, C.R. Acad. Sci. Paris Ser. I Math. 303 (1986), 923-927. MR 88g:47010

[BM] L. Bernal-González and A. Montes-Rodríguez, Non-finite-dimensional closed vector spaces of universal functions for composition operators, J. Approx. Theory 82 (1995), 375391. MR 96f:30034

[Bi] G. D. Birkhoff, Démonstration d'un théorème élémentaire sur les fonctions entières, C. R. Acad. Sci. Paris 189 (1929), 473-475.

[Bo] R. Bouldin, The essential minimum modulus, Indiana J. Math 30 (1981), 513-517. MR 82i:47001

[Bou] P. S. Bourdon, Invariant manifolds of hypercyclic vectors, Proc. Amer. Math. Soc. 118 (1993), 845-847. MR 93i:47002

[BS1] P. S. Bourdon and J. H. Shapiro, Cyclic composition operators on $H^{2}$, Proc. Symp. Pure Math. 51 Part 2 (1990), 43-53. MR 91h:47028

[BS2] P. S. Bourdon and J. H. Shapiro, Cyclic phenomena for composition operators, Mem. Amer. Math. Soc. 596 (1997). MR 97h:47023

[CS] K. C. Chan and J. H. Shapiro, The cyclic behavior of translation operators on Hilbert spaces of entire functions, Indiana Univ. Math. J. 40 (1991), 1421-1449. [MR 92m:47060

[Co] J. B. Conway, A Course in Functional Analysis, Springer-Verlag, New York, 1985. MR 86h:46001

$[\mathrm{CM}] \quad$ C. C. Cowen and B. D. MacCluer, Composition Operators on Spaces of Analytic Functions, CRC Press, 1995. MR 97i:47056

[Di] J. Diestel, Sequences and Series in Banach Spaces, Springer-Verlag, New York, 1984. MR 85i:46020

[En] P. Enflo, On the invariant subspace problem for Banach spaces, Acta Math. 158 (1987), 213-313. MR 88j:47006

[GS] R. M. Gethner and J. H. Shapiro, Universal vectors for operators on spaces of holomorphic functions, Proc. Amer. Math. Soc. 100 (1987), 281-288. MR 88g:47060

[GoS] G. Godefroy and J. H. Shapiro, Operators with dense invariant cyclic vector manifolds, J. Funct. Anal. 98 (1991), 229-269. MR 92d:47069

[Ha] P. R. Halmos, A Hilbert space problem book, Van Nostrand Company, Inc, 1967. MR 34:8178

[He1] D. A. Herrero, Approximation of Hilbert space operators, vol. I second edition, Pitman Res. Notes Math., vol. 224, Longman Scientific and Technical, 1989. MR 91k:47002

[He2] D. A. Herrero, A metatheorem on similarity and approximation of operators, J. London Math. Soc. 42 (1990), 535-554. MR 92h:47021

[He3] D. A. Herrero, Limits of hypercyclic and supercyclic operators, J. Funct. Anal. 99 (1991), 179-190. MR 92g:47026

[HW] D. A. Herrero and Z. Wang, Compact perturbations of hypercyclic and supercyclic operators, Indiana Univ. Math. J. 39 (1990), 819-830. MR 91k:47042

[Ki] C. Kitai, Invariant Closed Sets for Linear Operators, Thesis, Univ. Toronto, 1982.

[LM] F. León-Saavedra and A. Montes-Rodríguez, Linear structure of hypercyclic vectors, J. Funct. Anal. 148 (1997), 524-545. MR 98h:47028b

[MZ] E. Makai and J. Zemánek, The surjectivity radius, packing numbers and boundedness below of linear operators, Integral Equations Operator Theory 6 (1983), 372-384. MR 84m:47005

[Mc] G. R. MacLane, Sequences of derivatives and normal families, J. Analyse Math. 2 (1952), 72-87. MR 14:741d

[Mo] A. Montes-Rodríguez, Banach spaces of hypercyclic vectors, Michigan Math. Journal 43 (1996), 419-436. MR 98g:47027

[Pa] M. Pavone, Chaotic composition operators on trees, Houston J. Math. 18 (1992), 47-56. MR 93c:47038 
[Re] C. Read, A solution to the invariant subspace problem on the space $\ell^{1}$, Bull. London Math. Soc. 17 (1985), 305-317. MR 87e:47013

[Ro] S. Rolewicz, On orbits of elements, Studia Math 32 (1969), 17-22. MR 39:3292

[Sa1] H. N. Salas, A hypercyclic operator whose adjoint is also hypercyclic, Proc. Amer. Math. Soc. 112 (1991), 765-770. MR 91j:47016

[Sa2] H. N. Salas, Hypercyclic weighted shifts, Trans. Amer. Math. Soc. 347 (1995), 993-1004. MR 95e: 47042

[Sh] J. H. Shapiro, Composition Operators and Classical Function Theory, Springer-Verlag New York, 1993. MR 94k:47049

[Shi] A. L. Shields, Weighted shift operators and analytic function theory, Topics in Operator Theory, Math. Surveys Monographs, vol. 13, Amer. Math. Soc., 1974, pp. 49-128. MR 50:14341

[SW] W. P. Seidel and J. L. Walsh, On Approximation by Euclidean and non-Euclidean Translates of an Analytic Function, Bull. Amer. Math. Soc. 47 (1941), 916-920. MR 4:10a

[Ze1] J. Zemánek, Geometric interpretation of the essential minimum modulus, in: Invariant Subspaces and Other Topics (Timisoara/Herculane, 1981), Operator Theory: Adv. Appl, vol. 6, Birkhäuser, 1982, pp. 225-227.

[Ze2] J. Zemánek, The semi-Fredholm radius of a linear operator, Bull. Polon. Polish Acad. Sci. Math. 32 (1984), 67-76. MR 86b:47020

Departamento de Análisis Matemático, Facultad de Matemáticas, Avenida Reina Mercedes, Apartado 1160, Sevilla 41080, Spain

E-mail address: fleon@cica.es

Departamento de Análisis Matemático, Facultad de Matemáticas, Avenida Reina Mercedes, Apartado 1160, Sevilla 41080, Spain

E-mail address: amontes@cica.es 